\title{
Long-term results of treatment of idiopathic blepharospasm with botulinum toxin injections
}

\author{
JOHN S ELSTON \\ From the Institute of Ophthalmology, Moorfields Eye Hospital, City Road, London, ECIV 2PD
}

SUMMARY One hundred and one patients with idiopathic blepharospasm have been treated with injections of botulinum toxin $A$ into the orbicularis oculi. Ten had previously had facial nerve avulsions and responded well, normal visual function being restored in the majority (7/10) for an average of 14 weeks. Without prior surgical treatment the response was more variable, but 71/91 regained normal or near normal vision. Older patients, those with a family history of the condition, and those without oromandibular dystonia responded slighly better. The severity of the blepharospasm, the length of the history, and spontaneous resolution of an episode of focal dystonia in the past had no influence on the outcome. Results were poor in the presence of an associated neurological disorder. Side effects, particularly a temporary partial ptosis, were common but were well tolerated. The average duration of improvement was eight weeks in men, nine in women, and there was no evidence of any increase in duration after multiple injections. Eighty nine patients continued with injections, 11 opted for surgical treatment, and one resumed drugs.

Botulinum toxin $\mathrm{A}$ is a dichain protein, with molecular weight 140000 , one of seven antigenically distinct neurotoxins produced by Clostridium botulinum, and a cause of the clinical syndrome of botulism, a progressive flaccid paralysis of muscle that follows ingestion of the toxin. ${ }^{1}$ It can be purified and accurately quantitated, and, because of its specific binding to acceptors on motor nerve terminals, local injection of very small doses of the toxin produces local paralysis, and individual muscles can therefore be selectively weakened. ${ }^{2}$ It interferes with the uptake of cytoplasmic acetylcholine by the presynaptic vesicles in the motor end-plate, and the duration and extent of the paralysis are dependent on the dose injected. ${ }^{34}$ Neuromuscular function is restored when new junctions are formed by sprouting from the axon, a process that takes six to eight weeks in the experimental animal. ${ }^{56}$

A pharmacological treatment for strabismus was developed on this basis in the late 1970's' and subsequently extended to idiopathic blepharospasm. ${ }^{78}$ At the dose used, no systemic immune response is encountered, ${ }^{9}$ and the treatment can be repeated indefinitely.

Correspondence to Mr J S Elston.

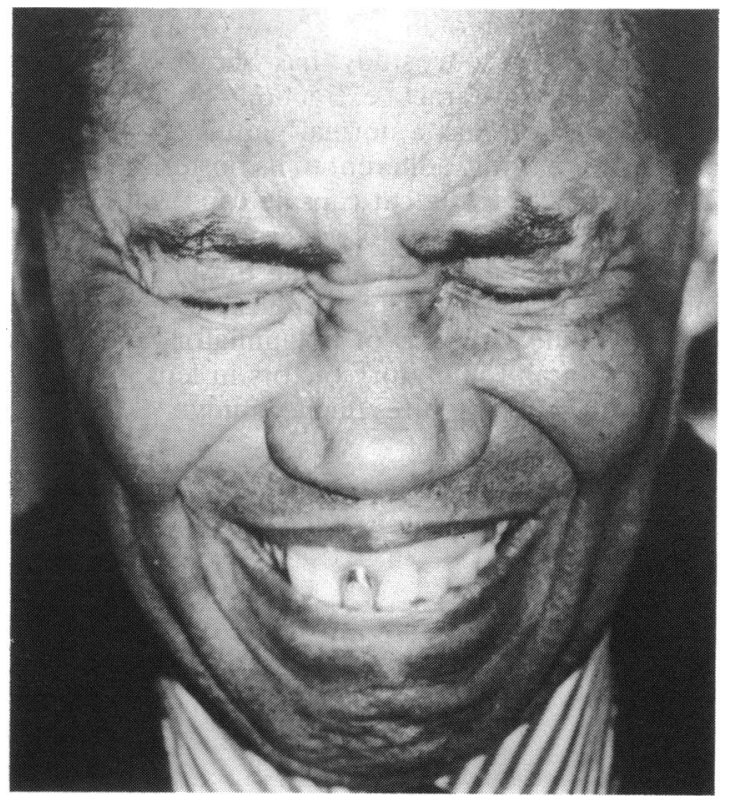

Fig. 1 Blepharospasm with oromandibular dystonia. The mouth is forced open and the jaw pulled down by spasm. 


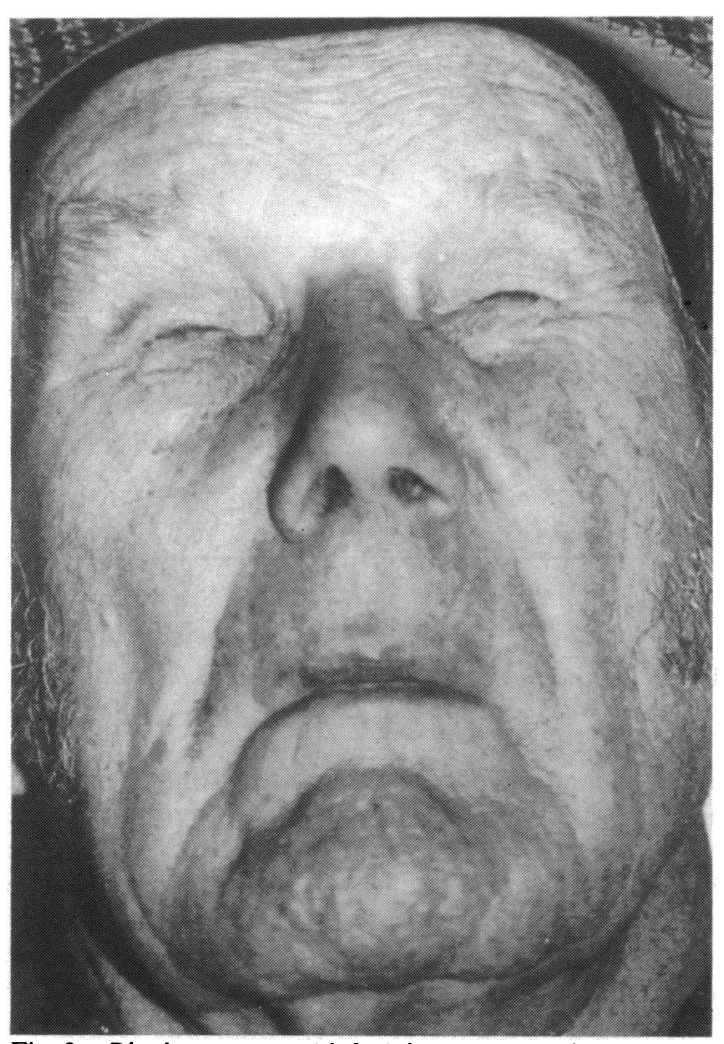

Fig. 2 Blepharospasmwith facial grimacing. The patient associates the grimace with an effort to open the eyes.

Blepharospasm is characterised by repeated involuntary spasms of forceful lid closure, progressing to functional blindness, and may be associated with spasms of the muscles of the lower face and jaw (oromandibular dystonia, Fig. 1) or facial grimacing on attempted eye opening (Fig. 2). It usually begins insidiously in the 5th to 7th decade, and is probably due to organic dysfunction of the basal ganglia." Because of the disfiguring nature of the condition, and frequently diagnostic delays, anxiety and depression are often associated. Blepharospasm may also occur in extrapyramidal disorders such as Parkinsonism and progressive supranuclear palsy.

Drug treatment is usually ineffective, although benzhexol may be useful." Denervation of the orbicularis oculi by facial nerve avulsion relieves the spasm, but the recurrence rate is $25 \%$ and upper facial mobility is abolished. ${ }^{12}$ The combined orbicularis stripping and local denervation procedure may also be followed by recurrent spasm..$^{13}$

The results in 101 cases treated with repeated injections of botulinum toxin, with a follow-up period of from 10 to 36 months, are presented in this report.

\section{Patients, materials, and methods}

Idiopathic blepharospasm was diagnosed clinically in 101 patients, 37 men and 64 women. The mean age at the onset of symptoms was 56.9 (range 26-74) and somewhat older in women $(58 \cdot 5)$ than men $(55 \cdot 3)$. Thirty-two had blepharospasm alone, 69 also had oromandibular dystonia, and, of these, 22 also had involuntary movements extending into the neck muscles which in eight interfered with eating or breathing. In 78 patients both with and without oromandibular dystonia attempts to open the eyes were associated with stereotyped facial grimacing and mouth opening. Five patients also had writer's cramp, three Parkinson's disease, two progressive supranuclear palsy, one tardive dyskinesia, and one generalised dystonia. There was a family history of focal dystonia in first-degree relatives in seven cases and in five an episode of focal dystonia had resolved spontaneously in the past (Table 1).

Most of the patients had been treated with a range of drugs without improvement, and 10 had had facial nerve avulsions, nine by one surgeon $(22.5 \%$ of the total operations performed). In four cases the surgery had been repeated (in two twice), and a further three patients had had orbicularis stripping operations (one twice) and one stereotactic neurosurgery. The patient's visual disability was assessed on a functional scale, and graded from 1 to 6 (Table 2 ), and the same scale was used to assess the effects of treatment.

Botulinum toxin $\mathrm{A}$ in ampoules containing $0 \cdot 05 \mu \mathrm{g}$ freeze-dried neurotoxin was dissolved in $10 \mathrm{ml}$ of sterile saline; the initial treatment was $1.6 \mathrm{ml}$ of this

\section{Table 1 Clinical features of patients treated}

Total patients: 101

Age at onset:

Female 64

Oromandibular dystonia

Facial grimacing

Neurological disease

Parkinson's disease

Progressive supranuclear

palsy (PSP)

Tardive dyskinesia

Gencralised dystonia

Family history of focal dystonia

Other focal dystonia

Writer's cramp

Torticollis or retrocollis

Previous resolved episode

of focal dystonia

Previous treatment drugs

Facial nerve avulsion

Orbicularis stripping

Stereotactic neurosurgery average $56 \cdot 9$ ycars (range 26-74) Male 37

$68 \cdot 3 \%$ $78 \%$

7 patients (all malc $=19 \%$ of $\mathrm{men}$ ) 3

2

1

7

9

5

4

4

93

10

3 
Table 2 Scale used for assessment of visual function before and after treatment

\begin{tabular}{|c|c|}
\hline Category & Visual function \\
\hline $\begin{array}{ll}1 & \text { Blind } \\
2 & \text { Dependent } \\
3 & \text { Independent }\end{array}$ & $\begin{array}{l}\text { Unable to see at all } \\
\text { Needs assistance outside home environment } \\
\text { Poor function: can go out alone, cannot read } \\
\text { or watch TV }\end{array}$ \\
\hline 4 Independent & $\begin{array}{l}\text { Moderate function, can read a little, cannot } \\
\text { work }\end{array}$ \\
\hline $\begin{array}{l}5 \text { Inconvenienced } \\
6 \text { Normal }\end{array}$ & $\begin{array}{l}\text { Able to work, cannot drive a car } \\
\text { Full-time normal activity - working, driving }\end{array}$ \\
\hline
\end{tabular}

solution, $0 \cdot 2 \mathrm{ml}$ laterally and medially in the upper and lower lids. Half this dose was used in patients who had had facial nerve avulsion. The treatment was an outpatient procedure; patients were reexamined after two weeks and therefore at monthly intervals, or sooner if the spasm returned. Repeat injections are of between $50-75 \%$ of the initial dose, and all patients have received at least three separate sets of injections.

\section{Results}

After a latent period of 24 to 48 hours the intensity of the blepharospasm progressively lessened over 5 to 6 days. Seventy-eight patients were either completely relieved or only inconvenienced to a minor extent by residual spasms (Fig. 3). Of the remainder, four remained functionally blind or dependent due to a continuing Bell's phenomenon elevating the eyes behind open lids. Eight had only minimal

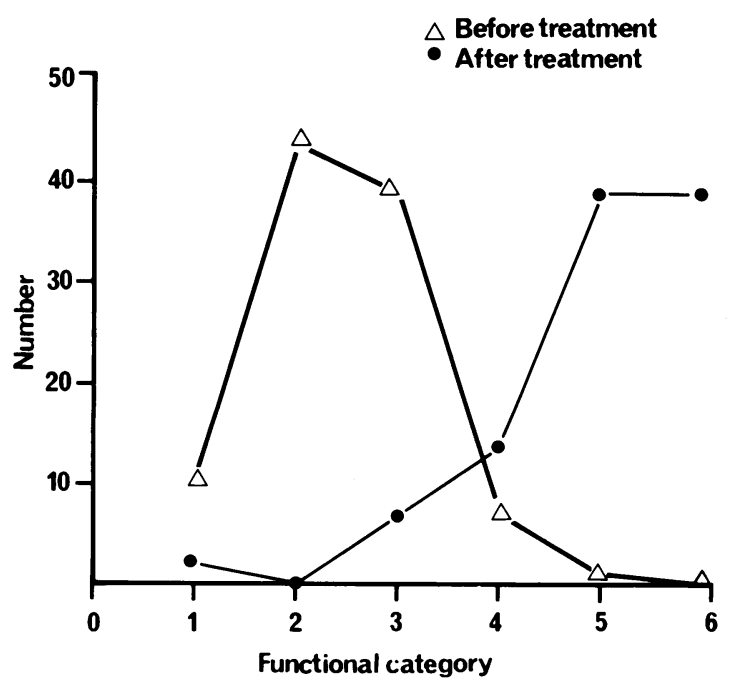

Fig. 3 Response to treatment in 101 patients with idiopathic blepharospasm.

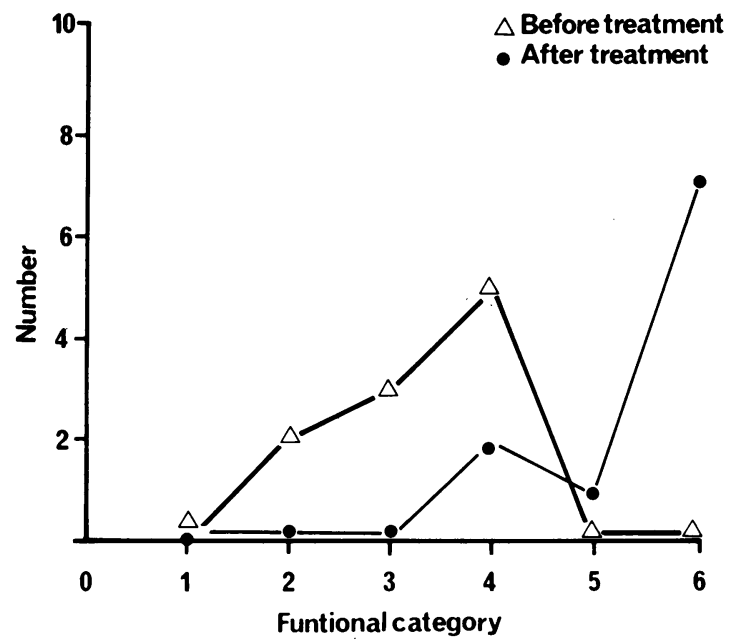

Fig. 4 Response to treatment in 10 patients who had previously had facial nerve avulsion.

improvement, while 11 were given some degree of independence. After a facial nerve avulsion there was generally a good result (Fig. 4), but orbicularis stripping had no influence on the response. Of the remainder, older patients had a marginally better response, as did those with no oromandibular dystonia or with a family history of focal dystonia. The length of the history or spontaneous resolution of an episode of dystonia in the past had no influence. In the presence of generalised neurological disease the results were poor.

Symptomatic improvement or relief lasted on average nine weeks, appreciably longer after a facial nerve avulsion (14 weeks); women had a slightly better sustained response than men (nine weeks compared with eight), but no other variable feature, such as age, severity, or length of history had any influence.

Sixteen of the 69 patients with oromandibular dystonia found that their facial movements were reduced or abolished when the blepharospasm was relieved, while facial grimacing associated with the effort to open the eyes was always relieved when the upper face spasm was reduced.

The commonest side effect was lid swelling, occurring in most cases after each treatment and resolving in one to two weeks. A more severe ptosis with reduced levator function was also common, occurring every third or fourth injection in half the patients, but it was short lived and did not affect visual function (Table 3). Three cases of profound ptosis with extraocular muscle weakness due to inadvertant overdosage took up to seven weeks to recover fully, and in six other patients there were episodes of temporary diplopia due to either inferior 
Table 3 Temporary side effects

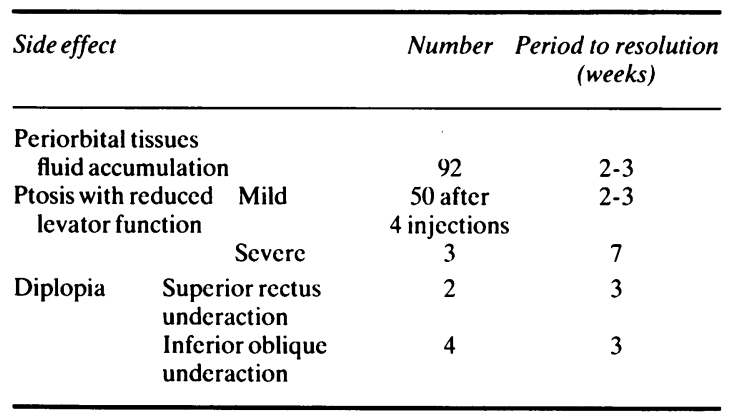

Table 4 Follow-up: long-term treatment

\begin{tabular}{lc}
\hline Treatment & No of patients \\
\hline Repeated injections with maintenance & 89 \\
$\quad$ of improvement & 1 \\
Resumption of systemic drug treatment & \\
Surgical treatment & 8 \\
Facial nerve avulsion & 3 \\
Orbicularis stripping & 4 \\
Resumption of injections after surgery & \\
\hline
\end{tabular}

oblique or superior rectus underaction. Although the dose of toxin needed to sustain the improvement diminished with repeated injections, the interval between treatments remained the same.

Eighty nine patients have continued with injection treatment, one has resumed drugs, and 11, after between three and five sets of injections, have had surgical treatment (Table 4). Four have subsequently had to resume injections owing to a recurrence. This group all had oromandibular dystonia and a poor or ill-sustained response to botulinum toxin but were neither more severely affected nor more subject to side effects.

\section{Discussion}

The clinical features of patients treated in this study were typical of idiopathic blepharospasm," "with onset in the 6th decade, a high incidence of oromandibular dystonia, and a female preponderance. Drug treatment had either not been successful or produced too many side effects. Within two weeks of the first injection $77 \%$ were substantially improved, with $39 \%$ functioning normally. Although the duration of symptomatic relief was only nine weeks, there was detectable weakness of the orbicularis oculi for much longer, and the muscle probably has to be reduced to less than $90 \%$ of its normal strength to allow the eyes to stay open. ${ }^{14}$ The dose needed to sustain this degree of weakness of the orbicularis declines with successive injections, but there is no increase in the

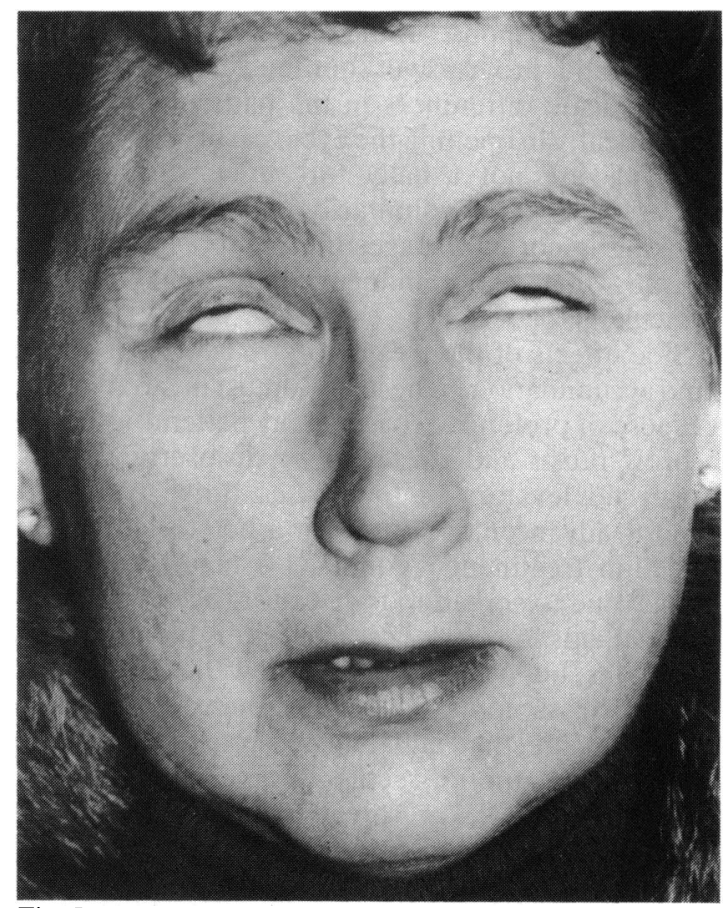

Fig. 5 Persistent Bell's phenomenon after treatment.

Despite profound weakness of the orbicularis oculi, the patient remains functionally blind, as the eyes move up under the upper lids.

period of relief, and sprouting from the terminal axon must be continuing. After a facial nerve avulsion recovery of muscle power takes substantially longer, and, since the treatment makes the orbicularis no weaker than in unoperated cases, the regenerated axon must be less capable of sprouting.

Bell's phenomenon is a normal accompaniment of forceful lid closure, and presumably present in all patients before treatment. Four patients remained visually disabled by it after treatment (Fig. 5), so that in the remainder paralysing the orbicularis must have abolished the associated eye movement. In the same way oromandibular dystonia was indirectly relieved or improved in a substantial minority. The explanation is not clear. Motor activity elsewhere in the body - for example walking - often exacerbates blepharospasm and oromandibular dystonia, presumably by a central mechanism. Relief of one aspect of abnormal motor function by peripheral paralysis must influence related movements centrally. Facial grimacing, although sometimes described by the patient as a voluntary attempt to open the eyes, has dystonic features, being a stereotyped movement, often associated with torsional neck movements, and it is also always abolished if the eyes can be opened. 
The poor response in the presence of Parkinson's disease and progressive supranuclear palsy (PSP) may indicate differences in the pathophysiology of the difficulty in opening the eyes, particularly in PSP. The lids are not usually forcefully squeezed but rather cannot be voluntarily opened, and reflex blinking is normal, suggesting an apraxia of eyelid opening that is not influenced by weakening the orbicularis.

Side effects of the treatment are common. Tissue fluid accumulation in the lids is almost invariable, and because of prolonged spasm many patients also have a brow ptosis and stretching or disinsertion of the upper lid levator. In four cases brow fixation or levator advancement has been a helpful adjunct to injection treatment. A mild (1-2 $\mathrm{mm})$ asymmetric ptosis, due to spread to the levator, occurred in half the patients on one occasion after four or more injections, but was short lived and well tolerated. An episode of diplopia developing three or four days after the injection and due to spread of paralytic effect to the superior rectus (three cases) or inferior oblique (four cases) resolved within a maximum of three weeks, with the symptoms being controlled by prisms. A complete ptosis with external ophthalmoplegia occurred in three cases after an inadvertent 10 -fold overdose of toxin. All these cases recovered normal lid opening and ocular muscle function, but the eldest patient took up to seven weeks to do so. The blepharospasm returned after a further eight to 10 weeks, and all these patients have had at least two further sets of injections without incident.

The 11 patients who elected to have surgical treatment (Table 4) obtained little or very short lived relief from injections. They did not have an excess of side effects and were not distinguishable before treatment from those who responded well. In four cases with persistent Bell's phenomenon the problem remained after successful facial nerve avulsion, but it resolved after six weeks in two patients. Four of the 11 patients treated surgically (three facial nerve avulsions, one orbicularis stripping) have had to resume injections, and in the patient who had had muscle surgery subcutaneous scarring made this very uncomfortable.

\section{Conclusion}

Botulinum toxin treatment is a safe and effective way of controlling idiopathic blepharospasm, and at the same time in the majority of cases relieving facial grimacing. Associated dystonic movements may also be indirectly improved in a minority.

\section{References}

1 Larson HE. Botulism, gas gangrene and clostridium gastrointestinal infections. In: Weatherall DJ, Ledham, JGG, Warell DA, cds. Oxford textbook of medicine. Oxford: Oxford University Press, 1982: 230-7.

2 Scott AB. Botulinum toxin injection into extra-ocular muscles as an alternative to strabismus surgery. Ophthalmology 1980; 87: 1044-9.

3 Boroff DA, Del Castino J, Envoy WH, Stcinhardt RA. Observations on the action of botulinum toxin $A$ on the frog neuromuscular junction. J Physiol (Lond) 1974; 240: 227-53.

4 Duchen LW. An electron microscopic study of the changes induced by botulinum toxin in the motor end-plates of slow and fast skeletal muscle fibres in the mouse. J Neurol Sci 1971; 14: 47-160.

5 Duchen LW. Changes in the electron microscopic structure of slow and fast skeletal muscle fibres in the mouse after the local injection of botulinum toxin. $J$ Neurol Sci 1971; 14: 61-74.

6 Scott AB, Rosenbaum A, Collins CC. Pharmacological weakening of extra-ocular muscles. Invest Ophthalmol Vis Sci 1973; 12: 924-7.

7 Scott AB, Kennedy RA, Stubbs HA. Botulinum toxin injection as a treatment for blepharospasm. Arch Ophthalmol 1985; 103: $347-50$.

8 Elston JS, Ross-Russell RW. Effect of treatment with botulinum toxin on neurogenic blepharospasm. Br Med J 1985; 290: 1857-9.

9 Biglan AW, Gonnering R, Lockhart LB, Rabin B, Fuerste FH. Absence of antibody production in patients treated with botulinum toxin. Am J Ophthalmol 1986; 101: 232-5.

10 Jankovic J, Ford J. Blepharospasm and orofacial-cervical dystonia: clinical and pharmacological findings in 100 patients. Ann Neurol 1983; 13: 402-11.

11 Skarf B, Sharp JA. Choline for blepharospasm. N Engl J Med 1981; 305: 957-8.

12 Bird AC, MacDonald WI. Essential blepharospasm. Trans Ophthalmol Soc UK 1975; 95: 250-3.

13 Gillum WN, Anderson RL. Blepharospasm surgery: an anatomical approach. Arch Ophthalmol 1981; 99: 1056-62.

14 Freuh BR, Felt DP, Wonjo TH, Musch DC. Treatment of blepharospasm with botulinum toxin. Arch Ophthalmol 1984; 102: $1464-8$.

Accepted for publication 26 September 1986. 